\title{
Agua y turismo como instrumentos de acumulación de capital, el caso de Benidorm, España
}

Recibido: 07/03/2016 · Aceptado: 12/11/2016

Nadia Hernández Peñaloza*

Lilia Zizumbo Villarreal

Universidad Autónoma del Estado de México

Teresa Torregrosa Martí

Universidad de Alicante, España

\section{Resumen}

En el presente artículo se analiza de qué modo el agua y el turismo sirven como instrumentos de acumulación de capital, ya que se considera al turismo una actividad económica para cuyo funcionamiento es indispensable el uso del recurso hídrico, lo que se ve reflejado en el consumo de $1 \%$ de agua dulce a nivel mundial, para atender la demanda se ha requerido la captación y distribución en alta, desde los lugares de aprovisionamiento y las redes de distribución en baja, técnicas que difícilmente pueden ser subsidiadas en su totalidad por el Estado, por ello se concesiona a empresas privadas la gestión del agua para las actividades turísticas, esto llega a ocasionar que se beneficie a un solo sector y afecte a otras actividades económicas de las regiones donde se asienta el turismo. De esta manera se genera una acumulación de capital para el sector privado, gracias a la explotación del turismo.

Palabras clave: Benidorm, agua, turismo, acumulación de capital.

*Correos electrónicos: nadis_7@hotmail.com·lzv04@yahoo.com·teresa.torregrosa@ua.es 


\title{
Water and tourism as instruments for capital accumulation, the case of Benidorm, Spain
}

\author{
Nadia Hernández Peñaloza* \\ Lilia Zizumbo Villarreal \\ Universidad Autónoma del Estado de México \\ Teresa Torregrosa Martí \\ Universidad de Alicante, España
}

\begin{abstract}
The aim of this paper is to analyze how water and tourism serve as instruments of capital accumulation considering tourism as an economic activity for which operation the use of water resources is essential. This is reflected in the consumption of $1 \%$ of fresh water resources worldwide. To meet the demand alternatives have to be identified such as the collection and distribution on high, from the places of supply and distribution networks in low, techniques that can hardly be subsidized entirely by the state. Therefore, concessions on water management for tourism purposes have been granted to private businesses resulting in benefits for a single sector affecting other economic activities of the region. Thus an accumulation of capital through the exploitation of tourism is generated for the private sector.
\end{abstract}

KEY woRDs: Benidorm, water, tourism, capital accumulation.

*E-mail: nadis_7@hotmail.com·lzv04@yahoo.com·teresa.torregrosa@ua.es 


\section{Introducción}

A medida que el capitalismo se ha posicionado como el sistema económico dominante, la sociedad ha modificado sus necesidades; como consecuencia se han desarrollado sectores de la economía, como el terciario o de servicios que, según Romero, Toledo y Ocampo (2014), se considera como un importante generador de empleo y representa gran parte del producto interno bruto (PIB) en el nivel mundial tanto en economías desarrolladas como en las que se encuentran en desarrollo. Este sector presenta un crecimiento y especialización cada vez mayores, que van de la mano de la globalización. El turismo es un ejemplo de ello; de acuerdo con Cordero (2006), este tiene una participación en el ámbito global como una potencialidad económica, es decir, como perfeccionamiento y desarrollo de los medios de producción y los servicios que permiten llevar a cabo comunicaciones, alojamientos, la producción de alimentos y el entretenimiento, lo que a su vez contribuye a una demanda capaz de aumentar los ingresos cada año: el turismo genera en el mundo 1245000 millones de dólares que, en conjunto con los servicios indirectos del sector, suma $9.5 \%$ al PIB, 9 $\%$ de empleos y produce $5.4 \%$ de exportaciones en el nivel mundial. Dentro de la economía española contribuye con $10.9 \%$ al PIB, $12 \%$ de empleo y tiene una cobertura de $276 \%$ del déficit comercial. Específicamente Benidorm, por actividades turísticas, produce 127 millones de euros al año, con una contribución de $6 \%$ al PIB, en el ámbito municipal, y 43 \% del PIB de la Comunidad Valenciana (Observatorio Turístico de la Comunidad Valenciana, 2014; Ministerio de Industria, Energía y Turismo, 2015; World Travel \& Tourism Council, 2015; Organización Mundial del Turismo [омт], 2015).

Se considera al turismo como una actividad económica capaz de generar empleos y un aumento en las economías de los países, pero también es una actividad segregada que no produce ninguna relación con las comunidades locales (Hiernaux, 2002); a pesar de ello, es vista como una alternativa de desarrollo que, en algunos países tanto desarrollados como subdesarrollados, es la principal fuente de ingresos.

La actividad turística requiere incorporar los bienes naturales y culturales de los pueblos como atractivos para el desarrollo de la misma, además de una estructura que permita ofrecer al visitante las condiciones para satisfacer sus necesidades de alojamiento, alimentación y ocio. Con este panorama resulta 
indispensable mantener tal actividad mediante la disposición, uso y control del recurso denominado agua.

Una vez que se combina la actividad turística con el uso del recurso agua, se generan las condiciones que permiten desarrollarla, sin embargo, si bien el turismo surge como una alternativa para las economías de los diferentes países, también es utilizado como estrategia para la acumulación del capital. De manera que, quien tiene el control del recurso hídrico puede controlar la actividad turística o cualquier otra actividad económica que requiera agua para su funcionamiento, y obtener ganancias que benefician generalmente al sector privado.

El propósito del presente artículo es explicar cómo se da una acumulación de capital por parte de la propiedad privada, mediante el control del uso del agua para la actividad turística, delineando el desarrollo de los destinos turísticos, así como la limitación de la población local de crecimiento económico y mejores condiciones de vida.

Esto se pretende explicar a través del turismo y el uso del agua, en el caso específico del municipio de Benidorm, elegido por ser un destino que prácticamente fue planeado para el desarrollo del turismo, que ha convertido el recurso agua en el elemento principal para su funcionamiento. Es aquí donde claramente se identifica la propiedad privada del recurso, por parte del sector hotelero; este caso demuestra que existe una acumulación capitalista a partir del turismo.

\section{Metodología}

El presente artículo se aborda desde la perspectiva de la dinámica de acumulación que parte del proceso de desposesión que, apoyada por el neoliberalismo, va en crecimiento afectando a poblaciones de la escala global.

En este caso se aborda desde la variable de privatización y mercantilización, la cual supone una transferencia de activos de la esfera pública a los dominios de lo privado con privilegios de clase (Harvey, 2005). Esta variable se utiliza específicamente en el caso del agua.

En un sentido más amplio la acumulación por desposesión no solo se refiere a la mercantilización de los bienes naturales, sino también a los bienes comunes sociales que pueden ser privatizados, es decir, que además de operar destruyendo las formas de propiedad comunal o público estatal, también las 
que están a favor de la propiedad capitalista transnacional o local asociada al círculo de valorización (Seoane, 2013).

Por su parte, también se incluye la participación del Estado como entidad política que organiza los procesos de acumulación (Lengyel, 1997) ya que precisamente es quien diseña políticas que lo convierten de una entidad de seguridad competitiva en una cada vez más limitada por la lógica del capital.

\section{El turismo como actividad económica}

El turismo es una actividad económica que ha experimentado un desarrollo expansivo, con el que ha adquirido importancia en el ámbito mundial. Los ingresos derivados del gasto de los turistas internacionales tanto en hospedaje como en alimentos y bebidas, entretenimiento y servicios complementarios son aproximadamente de 1245000 millones de dólares, según la омт (2015). Los principales destinos se encuentran en Estados Unidos, cuya derrama económica suma 48000 millones de dólares; Europa, con 509000 millones de dólares; Asia y el Pacífico, con 377000 millones de dólares; América, con 274000 millones de dólares; Oriente Medio, con 49 000, y África, con 36000 millones de dólares.

A lo largo del tiempo, esta actividad ha generado un incremento acelerado de turistas: a escala mundial las llegadas de visitantes internacionales han registrado un crecimiento de 25 millones en 1950, a 278 millones en 1980, 527 millones en 1995 hasta 1133 millones en 2014. Por ello, cada vez más lugares están impulsando la actividad turística, ya que según la omT (2015), actualmente la cuota de mercado de este sector en las economías emergentes ha aumentado de $30 \%$ en 1980 a $45 \%$ en 2014, por lo que se espera que para el año 2030 crezca en $57 \%$.

En este sentido, la омт (2015) menciona que un número creciente de destinos en todo el mundo se han abierto al turismo, consolidando así un sector clave para el progreso socioeconómico, a través de la creación de empresas, empleos, generación de ingresos de exportación y ejecución de infraestructuras. Asimismo, la Organización de las Naciones Unidas (2013) menciona que muchos países consideran al turismo como una actividad importante para el progreso económico y la reducción de la pobreza, pero esto depende de que el turismo genere oportunidades de empleo, cree eslabonamientos con los demás sectores productivos y estimule el desarrollo de infraestructura básica, es decir, 
que beneficie a la economía en su conjunto. Además de que el desarrollo del turismo sea conducido por estrategias nacionales que prevean políticas y marcos reguladores, así como instituciones que estimulen el desarrollo de la oferta en los mercados y reduzcan los efectos negativos generados.

Sin embargo, en este ámbito de crecimiento global y para generar tales ingresos, de acuerdo con Dotres (2003), se ha tenido que priorizar la privatización, trayendo inversionistas y empresarios nacionales y extranjeros para el sostenimiento de la actividad, ignorando los efectos ambientales y las consecuencias que se originan a partir de la expansión del turismo. Entonces, el turismo es una actividad económica que, como señala Vera (1986), crea conflictos por el uso del suelo, el agua y los recursos naturales en general, además de una disputa por la dominación del territorio; produce un cambio en el modelo económico y renueva las actividades económicas predominantes. El turismo es un catalizador para la expansión del capital, mediante inversiones en infraestructura que atienden las demandas de los visitantes, como aeropuertos, carreteras, hoteles, entre otros (Palafox, Zizumbo y Arriaga, 2010), que aparecen en territorios con recursos tanto naturales como culturales, con la intención de atraer ingresos económicos, aunque no necesariamente en beneficio de las poblaciones receptoras.

\section{Turismo en Benidorm}

Benidorm es un municipio ubicado en la provincia de Alicante, España; forma parte de la Comunidad Valenciana y de la ribera del mar Mediterráneo. Está situado dentro de la Comarca de la Marina Baja (Ordoñez y Rovira, 2011). Su superficie física total es de 3817 ha, y tiene una superficie urbanizada de 1629 ha, y $14.3 \mathrm{~km}$ de litoral; su temperatura promedio anual es de $18.64^{\circ}$ (Ayuntamiento de Benidorm, 2014).

En 1950, Benidorm tenía 2726 habitantes de los cuales 60 \% vivía del sector productivo primario, que solo contaba con 642 viviendas y la densidad de la población era de $10 \mathrm{~m}^{2}$ por habitante; era un lugar de calles estrechas donde no existían avenidas ni paseos importantes y la actividad comercial era muy escasa. Dada la crisis que atravesaba el sector primario se impulsó un cambio de orientación económica hacia el sector turístico, gracias a los recursos con los que contaba: para 1953 comenzó la planeación urbanística de la ciudad, 
que implicó un crecimiento habitacional y con ello la economía comenzó a recuperarse (Higueras, 2012). En los años posteriores, la población aumentó de 6202 habitantes en la década de los sesenta, a 12547 habitantes; en los años ochenta había 24783 habitantes, en los noventa 57 227, para el año 2010 había un total de 75948 habitantes. En la actualidad el municipio tiene 69010 habitantes (Ayuntamiento de Benidorm, 2014; Instituto Nacional de Estadística, 2015). Lo anterior refleja un fuerte crecimiento que demanda muchos servicios, entre los cuales destaca una mayor demanda de agua potable: el consumo anual es de $10002550 \mathrm{~m}^{3}$ (Ayuntamiento de Benidorm, 2014).

De acuerdo con investigaciones de Mazón (2010), Benidorm se caracterizaba por ser un pequeño pueblo cuya población se dedicaba principalmente a la pesca y la agricultura, sus playas eran aprovechadas por familias adineradas del interior de la provincia que llegaban al lugar buscando tranquilidad y disfrutar las propiedades curativas del agua del mar. En ese entonces, solo había dos posadas y una pensión; más tarde, en la década de los treinta, se edificó el primer hotel llamado Bilbaíno; se construyeron también los primeros chalets, que con los años y el crecimiento urbano desaparecieron. Como los visitantes iban en aumento y solo se contaba con la infraestructura de hospedaje mencionada, algunos pobladores del lugar rentaban sus viviendas en épocas vacacionales, pues se trasladaban a sus huertas, y de esta manera obtenían ingresos extras.

Puede decirse que a partir de 1950 comienza el desarrollo del turismo nacional de masas con la aprobación del ordenamiento urbanístico de la villa en 1953 cuya finalidad era crear una ciudad concebida para el ocio y el turismo; para 1959 se abren cuatro nuevos hoteles; en 1959 se inaugura el festival de la canción y se permite el uso del bikini en las playas; en 1962 se edifica la plaza de toros; en 1963 se construye el segundo plan general que aumenta la altura de los rascacielos y propone una densidad habitacional menor; en 1964 se celebra la primera asamblea nacional de turismo; en 1970 se abre el aeropuerto de Alicante; en 1975 se construye la autopista que comunica a Alicante con Benidorm; en 1978 comienza una escasez de agua (a la que se le hizo frente mediante la cesión de 70 millones de pesetas por parte del Estado); en 1985 se abre el parque temático Aqualandia; en 1987 se obtiene la bandera azul concedida por la Unión Europea (certificación de limpieza y seguridad); para el 2000 se abre el parque temático Terra Mítica, Aqua Natura y Terra Natura. En 2005 se renuevan 
hoteles y aumentan de categoría; se construye un campo de golf (Espinosa, 2011; Ordoñez y Rovira, 2011); estos últimos acontecimientos aportaron un plus al desarrollo de la actividad turística.

A la par de tales acontecimientos, según Higueras (2012), en los años 1978 y 1979 una gran sequía estuvo a punto de acabar con el modelo turístico de la ciudad; se hablaba de que en Benidorm únicamente pensaban en construir, sin considerar el agua, pero una serie de obras permitió encontrar acuíferos superficiales y subterráneos e instalar bombeos y depuradoras; así el municipio consiguió autoabastecerse. Una vez resuelto el problema del agua, era primordial enfocarse al turismo; de acuerdo con Mazón, Delgado y Hurtado (2011), mediante el apoyo político se crearon condiciones para facilitar inversiones a grandes turoperadores y empresas intermediarias del turismo internacional, a través de créditos y fuertes inversiones para la construcción de complejos hoteleros y departamentos turísticos que impulsaron la actividad turística.

En la actualidad, la actividad económica de Benidorm se centra de manera casi exclusiva en el turismo de masas: al año es visitado por más de seis millones de turistas que, junto con el turismo residencial, suman aproximadamente 60 millones de pernoctaciones en ese periodo (Mazón, Delgado y Hurtado, 2011). En conjunto con los demás servicios de la oferta turística, en el año 2014 se generó una derrama económica de 127 millones de euros (Observatorio Turístico de la Comunidad Valenciana, 2014) contribuyendo de manera significativa con el PIB tanto de la Comunidad Valenciana, como al de España.

La oferta turística de este lugar, de acuerdo con el H. Ayuntamiento de Benidorm (2014), es de 128 hoteles, en su mayoría de tres estrellas; nueve pensiones; tres hostales; diez campings y, los más representativos, los departamentos, que suman 5 939; en cuanto a los establecimientos de alimentos y bebidas se registran 275 restaurantes, 128 cafeterías, 145 disco-pub y 576 bares; respecto a la recreación se ofertan 25 discotecas, 31 salones de juegos, tres bingos, dos cines, 14 salas cinematográficas, cinco juegos infantiles, dos parques acuáticos, un circo y ocho parques temáticos; las agencias de viaje registradas son 59.

Es importante mencionar que, a pesar de que existe oferta turística complementaria, el rubro de alojamiento, de 70190 plazas, es el que acapara el turismo, ya que en su mayoría los establecimientos ofrecen paquetes todo incluido, por ello los turistas únicamente salen para tomar el sol en la playa y regresan 
a tomar los alimentos en los hoteles, dejando el municipio casi desierto por las tardes.

\section{El agua como elemento esencial del turismo}

$\mathrm{El}$ agua se considera un recurso natural renovable con diversas funciones tanto económicas como sociales y naturales, sin embargo, en las últimas décadas se ha generado una preocupación internacional que se ha reflejado desde la Conferencia Internacional sobre el Agua y el Medio Ambiente, celebrada en Dublín en 1992 (Candanedo, 1992), hasta la Conferencia de Alto Nivel Internacional sobre la Implementación del Decenio Internacional para la Acción "el agua es fuente de vida” en 2015 (Departamento de Asuntos Económicos y Sociales de Naciones Unidas, 2015); tal preocupación radica en que el agua es requerida por pequeñas comunidades, grandes urbes, para actividades productivas, en la industria, pero sobre todo, en el turismo. Por lo tanto, el uso del agua cada día va en aumento gracias a la creciente demanda tanto de los sectores productivos, de servicios, como de las propias comunidades.

Como ya se mencionó, el agua es un instrumento principal con el que trabaja el turismo tanto para las actividades de ocio (spas, clubs de golf, balnearios, entre otros) para actividades recreacionales (sol y playa, ríos, arroyos, cascadas, termas, entre otros), para las empresas dedicadas a los servicios (hoteles, restaurantes, bares) como para todos los servicios complementarios (lavanderías, albercas, lavapiés, regaderas, entre otros), por lo que Rico (2007) señala que la disponibilidad de agua condiciona la oferta de productos y servicios turísticos: si un lugar turístico tiene problemas de escasez de agua, es posible que no pueda seguir funcionando en el largo plazo.

El consumo doméstico promedio de agua es de 100 litros por habitante por día (Saladié, 2013), esta cantidad casi se duplica en países desarrollados y en países menos desarrollados es de aproximadamente 20 litros por habitante por día; estas cifras dependen de cada país, pero en el nivel mundial es un promedio. Los destinos turísticos que reciben visitantes cada año en periodos vacacionales aumentan en cantidades grandes el consumo de agua, esto únicamente en consumo doméstico, es decir, para actividades básicas tales como preparación 
de alimentos, aseo personal, entre otros. Por lo que, el consumo de agua es aún mayor con la realización de actividades recreativas como las mencionadas.

Por ello, el agua es cada vez más escasa en el mundo (Petrella, 2011); debido a la lógica de desarrollo a cualquier precio, medido en función de los productos y servicios consumidos, aumenta la explotación de los recursos hídricos, sin importar el impacto que esto genere.

Por otro lado, además de la escasez de agua, un problema a partir de su uso es la apropiación del recurso: si bien se considera como un derecho humano al que todos debieran tener acceso, no es así, porque se encuentra en manos del Estado, que, según Witker (2015), es quien tiene la función de regular el uso y aprovechamiento en función del interés público, orientado hacia objetivos de conservación, protección, equidad en el acceso y eficiencia en su uso y prevención de su monopolización. Asimismo, Jouralev (2003) menciona que el encargado de aplicar la legislación asigna el agua mediante el otorgamiento de permisos, autorizaciones, concesiones, derechos u otros instrumentos; controla las condiciones de devolución del agua al ecosistema después de su uso; evalúa proyectos; aprueba obras; entre otras funciones. Que, por el contrario, mediante el uso de las facultades con las que cuenta, maneja el agua de acuerdo con lo que mejor le conviene, hablando en términos monetarios, y que en diversos países, lejos de buscar un suministro equitativo, otorga concesiones a los grandes inversionistas, generando de alguna manera una privatización.

En cuanto al suministro de agua, afecta directamente cuando es otorgado en mayores cantidades a las grandes empresas turísticas, las cuales pueden seguir creciendo, mientras que las pequeñas, al no contar con el recurso, no pueden aumentar la oferta de sus servicios; con respecto a los atractivos que tienen que ver con agua, como yacimientos de aguas termales, espacios delimitados como áreas naturales protegidas, entre otras, son privatizados en beneficio del turismo.

\section{Instituciones reguladoras de la gestión del agua en España}

En la política de desarrollo económico, la gestión del agua se ha convertido en una parte fundamental, no solo para regular planes, sino para adoptar medidas que garanticen el recurso, para lo cual es necesario crear políticas, organismos e instituciones que se encarguen de regular los diversos usos del agua. 
En el nivel mundial existen organismos que, si bien no son exclusivos de la regulación del agua, intervienen de manera directa o indirecta en cuestiones que tienen que ver con tal recurso, como la Organización de las Naciones Unidas para la Educación, la Ciencia y la Cultura (Unesco), que se enfoca en el desarrollo sustentable, el aprovechamiento y gestión de los recursos naturales; la Organización de las Naciones Unidas para la Agricultura y la Alimentación (FAo), que interviene a través de la ordenación sustentable de los recursos naturales; el Consejo Mundial del Agua (wwc), que nace como una institución que afronta la problemática en torno al agua; la Asociación Global del Agua (GWP), que engloba a todas las agencias gubernamentales, instituciones públicas, compañías privadas, organizaciones profesionales y agencias de desarrollo multilateral involucradas en la gestión del agua con la finalidad de conformar un uso sustentable de los recursos hídricos (Torregrosa, 2009).

Dentro de la Unión Europea la gestión del agua se realiza a través de organizaciones públicas, mediante la Comisión Europea, específicamente con la Dirección General de Medio Ambiente, cuyo propósito es proteger, preservar y mejorar el ambiente para generaciones presentes y futuras, mediante políticas de protección de alto nivel (Comisión Europea, 2016); el Parlamento Europeo, en concreto la Comisión de Medio Ambiente, Salud Pública y Seguridad Alimentaria, es responsable del desarrollo sostenible, la política ambiental, la contaminación de aire, suelo y agua, la gestión y reciclaje de residuos, las sustancias y preparados peligrosos, los niveles de ruido, el cambio climático y la protección de la biodiversidad (Parlamento Europeo, 2016) y la Agencia Europea de Medio Ambiente (2016), organismo que ofrece información solida del medio ambiente, es la fuente principal de información para los responsables del desarrollo, aprobación, ejecución y evaluación de las políticas medioambientales.

En España, el Ministerio de Agricultura, Alimentación y Medio Ambiente (2015) tiene entre sus funciones la regulación de usos permitidos sobre los bienes de dominio público hidráulico, la cooperación internacional y participación pública mediante acuerdos, informar sobre el estado y calidad del agua, y evaluar los recursos hídricos; este Ministerio integra los organismos autonómicos formados por Comunidades Hidrográficas cuyo campo de acción está delimitado por el territorio; el municipio en estudio se encuentra en la Comunidad Hidrográfica del Júcar, que tiene como principales funciones elaborar el 
plan hidrológico de cuenca, así como dar seguimiento y revisión, administrar y controlar el Dominio Público Hidráulico, llevar a cabo el proyecto, construcción y explotación de las obras realizadas con fondos propios y las encomendadas por el Estado, las derivadas de convenios con otras comunidades, corporaciones locales y entidades públicas o privadas (Confederación Hidrográfica del Júcar, 2015).

Siguiendo en la línea de comunidades autónomas están las consellerías; para cuestiones hídricas, existe la Consellería de Agricultura, Pesca, Alimentación y Agua, que se encarga de garantizar la calidad de aguas de baño, crear normativas de saneamiento para aguas residuales, gestionar y supervisar proyectos, así como la participación pública, y la Consellería de Infraestructura, Territorio y Medio Ambiente, que regula lo referente a las actividades náuticas, calidad ambiental y costas (Generalitat Valenciana, 2015).

Como parte de las instituciones gestoras del agua en el plano local se encuentra el Consorcio de Agua de la Marina Baja (САмв), creada mediante la búsqueda de una solución a las sequías registradas desde 1969 en la Comarca de la Marina Baja. En 1977, después de varias reuniones realizadas en el Gobierno Civil y Diputación Provincial, y tras varios acuerdos y reuniones, se crea el Consorcio para Abastecimiento de Aguas y Saneamientos de la Marina Baja (Rico, 2010), organismo que "constituye una entidad jurídica pública local, de carácter asociativo e institucional regulado por estatutos. Está dotado de personalidad jurídica plena e independiente de la de sus miembros y su capacidad jurídica de derecho público y privado es tan amplia como lo requiera la realización de sus fines" (Consorcio de Agua de la Marina Baja, 2015). Este Consorcio está integrado por la Diputación Provincial de Alicante y la Confederación Hidrográfica del Júcar. Dentro de sus funciones están: estudio de las necesidades de abastecimiento de aguas y saneamiento; elaboración, en colaboración con la Confederación Hidrográfica del Júcar, de los planes de aprovechamiento de las aguas y saneamientos de la comarca; redacción de estudios, anteproyectos y proyectos que satisfagan tales necesidades; dar seguimiento a las solicitudes de concesiones o autorizaciones para el abastecimiento de agua, tratamientos y vertidos residuales; realización de obras e instalaciones para tratamientos de aguas, evacuación y depuración de esas instalaciones; explotación y conservación de las instalaciones y coordinar las actividades 
con las del Ministerio de Obras Públicas (Consorcio de Agua de la Marina Baja, 2015).

Esta última organización ha formulado una serie de acuerdos con los regantes para la cesión de aguas limpias a cambio de residuales depuradas, además de compensaciones económicas en infraestructuras agrarias. Según Torregrosa (2009) y Rico, Olcina y Baños (2014), estos acuerdos consisten en el uso conjunto del canal bajo del Algar para transporte de agua blanca desde el río Algrar-Guadalest hasta el embalse de Amadorio, los costes de mantenimiento y explotación de aguas depuradas son asumidos por el Consorcio, el intercambio de agua blanca del sistema Algar-Guadalest por agua depurada y desalada a coste cero y el importe aproximado de la colaboración, entre 450000 y 600000 euros al año, más obras financiadas por el Consorcio, esto para la comunidad de regantes de Canal Bajo del Algar; con respecto a la comunidad de regantes de Villajoyosa es el uso conjunto del embalse de Amadorio para almacenar hasta 1500 l/s de sobrantes de la cuenca del Algar-Guadalest, la construcción del embalse y la red de distribución de agua depurada, intercambio de agua blanca del embalse de Amadorio por agua depurada a coste cero y el importe aproximado de la colaboración, de 81500 euros por año más obras financiadas por el Consorcio.

Para la comunidad de regantes de Callosa d'en Sarrià, el Consorcio asume los costes de energía de los pozos de Sacos-Algar, de otros bombeos a regantes y al Ayuntamiento de Callosa d'en Sarrià, financiamiento de obras hidráulicas e infraestructura agraria, los pozos de Sacos-Algar con 700 l/s que garantizan durante periodos de sequía los caudales circulantes en el río Algar compartidos por los regantes y por el Consorcio, y por último, en las comunidades de regantes de La Nucía y Altea, derechos sobre el uso de los ríos Algar-Guadalest, conexión de las redes de riego con conducción de agua depurada del Canal Bajo del Algar y con la depuradora de Altea, durante los periodos de sequía, los regantes emplean agua depurada para evitar tensiones por el uso de agua blanca del Algar-Guadalest y obras financiadas por el consorcio (Torregrosa, 2009). 


\section{Turismo y agua como elementos de mercantilización y privatización para la acumulación de capital en Benidorm}

La estructura del turismo en el ámbito global deviene de los intereses de los organismos internacionales por reproducir el modo de producción capitalista (Palafox, 2013), de manera que forma parte de un proceso en el que se realizan transferencias de recursos de carácter público a privados, mediante una desposesión a partir de la mercantilización y privatización de la tierra y del agua (Harvey, 2005). Entonces, se participa en una dinámica de expansión que se ha ido diversificando mediante la incorporación de nuevas alternativas que demandan mayor cantidad de recursos.

Por lo anterior el turismo constituye una fuerza hegemónica de la economía política, capaz de dirigir la acción productiva, articulando los diversos niveles de poder (López y Marín, 2010), que van desde instituciones reguladoras a nivel internacional, hasta la participación de autoridades locales y municipales. El Estado es la institución más importante en el plano nacional, ya que en él recae la toma de decisiones, si bien los organismos internacionales promueven acciones y políticas en materia turística, es precisamente el Estado quien decide cómo y cuándo se llevan a cabo. Mendoza (2000) señala que el Estado asume un papel de legislador, con influencia y control en el aspecto económico mediante el estímulo, que lo posiciona como otorgante de concesiones o subsidios para que las empresas de la iniciativa privada destinen su capital en nuevas inversiones; además de la reglamentación y la protección.

El Estado posee gran influencia en el desarrollo del turismo que, generalmente y por cuestiones que mejor le convienen, tiene preferencia por el capital privado el cual, mediante las fuertes inversiones, desarrolla la actividad para propiciar un crecimiento económico que, aunque se refleja en el PIB, no beneficia a la comunidad local.

Además del turismo, el agua es también un elemento que se administra bajo la legislación del Estado, quien tiene la obligación de proveer el recurso a la población, al campo y a las empresas o usuarios finales que lo necesiten.

En España, de acuerdo con Sánchez, Rodríguez y Salas (2011), el abastecimiento de agua se efectúa mediante la gestión en alta, que consiste en llevarla desde grandes obras de captación hasta los depósitos de cabecera y la gestión en baja, que se realiza transportando el agua desde los depósitos de cabecera 
hasta los usuarios finales bajo la responsabilidad de corporaciones locales como municipios, mancomunidades o entidades provinciales, cuya función es el abastecimiento urbano, alcantarillado y depuración de aguas urbanas.

Más específicamente en Benidorm, el agua para la agricultura, antes de contar con la tecnología y los métodos avanzados de recolección de agua, se obtenía mediante el uso de pequeñas balsas de riego, cuyos depósitos no superaban los $100 \mathrm{~m}^{3}$ para regar fincas de menos de 5 ha; para los años setenta el riego se realizaba mediante inundación con reparto de aguas por tandas con una duración de varias semanas; a partir de los ochenta, el agua se reparte a presión a todos los regantes con una instalación de contador de agua y conexión a pie de parcela, y actualmente se cuenta con una planta depuradora puesta en marcha en el 2006 que desala el agua residual por medio de ósmosis inversa cuya capacidad es de $25000 \mathrm{~m}^{3}$ al día (Gil y Rico, 2015; Rico, 2015; Vera, 2006), que es utilizada para el regadío de 2000 ha y en el mantenimiento de parques, jardines y limpieza en general de calles y zonas residenciales.

El abastecimiento de agua para el uso urbano corre a cargo de la empresa privada Aquagest, que obtuvo la cesión de derechos de agua potable y aguas fecales en 1987 por parte del ayuntamiento de Benidorm y que actualmente sigue operando dentro del municipio (Amillo, 2013). De esta manera, el recurso agua ha dejado de ser público y se ha convertido en un bien utilizado como mercancía.

Para el espacio turístico y el espacio agrario de la Marina Baja es fundamental el uso del agua (Torregrosa, 2009), sin embargo, el primero mantiene ventaja sobre el segundo, ya que al ser el turismo la principal actividad económica de la región es prioridad que se abastezca de agua el espacio turístico-urbano en primer plano (Juárez, 1994). Esto no significa que la agricultura se quede sin agua, sino que a través de convenios establecidos con los agricultores que ya se detallaron anteriormente, se realiza un intercambio de aguas limpias por aguas depuradas.

El abastecimiento de agua para el año 2009 fue de $12855994 \mathrm{~m}^{3}$ para uso urbano y $3524965 \mathrm{~m}^{3}$ para riego (Vera, 2006). Esta situación de desventaja se da principalmente por el crecimiento turístico que se ha presentado, porque al aumentar la demanda turística también lo hace la demanda de agua, y el gobierno, al ver que el turismo es la actividad económica predominante y que la agricultura va en descenso, da prioridad a la primera, creando políticas y acuerdos para el aseguramiento del agua. 
Es claro que el espacio turístico, como actividad económica casi exclusiva del lugar, está por encima de la agricultura, pues genera 127000 millones de euros al año (Observatorio Turístico de la Comunidad Valenciana, 2014), entonces debiera suponerse que la calidad de vida del municipio es buena, pero ¿cuál es realmente la situación en Benidorm a causa de la propiedad privada del agua y su uso turístico?

Las estadísticas anuales muestran que $87.7 \%$ de empresas pertenece al sector servicios, de ellas $21 \%$ se dedica a hospedaje, y del $86.1 \%$ de empleos en el sector terciario, 35.6 \% corresponde a la hotelería (Mazón, 2010). Estas cifras muestran que el turismo es la principal fuente de ingresos del municipio, generando empleos directos e indirectos, sin embargo, la población local no se beneficia realmente, ya que solo participa dentro de la actividad como mano de obra y no como empresarios o dueños de los establecimientos.

Por lo tanto, los pobladores no pueden competir con los grandes infraestructuras hoteleras, ya que solo son empleados que trabajan con condiciones laborales pésimas ${ }^{1}$ (Mazón y Delgado, 2009), entre las cuales se describen los bajos salarios, empleos temporales sin seguridad social y jornadas excesivas, cuestiones que producen migración de la población originaria.

Por otro lado, como parte complementaria de la hotelería, se encuentra el turismo residencial que, por la falta de planificación y crecimiento acelerado, consume cada vez mayores espacios que modifican el paisaje y a su vez produce problemas ambientales como el tratamiento de desechos sólidos y la depuración de las aguas, masificaciones humanas y de tráfico, contaminación acústica, de aire, agua, suelo, visual, etc., sumadas a la reducción del espacio litoral.

Por otra parte, está la población que cuenta con pequeñas empresas dedicadas al área de alimentos y bebidas que, de igual forma, no compiten en el mercado gracias al todo incluido de los grandes hoteles a bajos costos, que incluso ofrecen sus servicios de alimentación a cualquier persona, no solo a los huéspedes, hecho que provoca que el lugar se vuelva desértico a la hora de los alimentos, y contribuye al cierre de los establecimientos.

Entonces, a través del turismo se produce un modelo de actuación en el que quienes controlan los hilos de la actividad son los grandes inversionistas

${ }^{1}$ Véanse entrevistas de los empleados del sector turístico de Benidorm en los Discursos Públicos de los Agentes Sociales de Benidorm, en Mazón y Delgado, 2009. 
que, de acuerdo con Gaviria (1976), crean una nueva fuente de acumulación de capital con la que claramente se puede explicar la monopolización e internacionalización de la exportación turística.

\section{Remunicipalización}

Con el modelo neoliberal, la política y la economía, como parte de las políticas públicas del Estado, han propugnado por que el sector público deje de prestar servicios de manera directa, es decir, que mediante diferentes formas de privatización, han propuesto que el mercado sea quien se encargue de proveer a la población de tales recursos. Sin embargo, cada vez más países se inclinan hacia la remunicipalización de la gestión de los servicios, entre ellos Alemania, Francia, Reino Unido y España. Lo anterior con la intención de ahorrar recursos o, como señala Hall (2011), por cuestiones de mala gestión por parte de las empresas, la expiración de contratos, reducción de costos, para una mayor eficiencia, para lograr objetivos, mejorar cuestiones laborales, etcétera.

La remunicipalización es el término que Wollmann (2013) define como el retorno a la gestión pública de la propiedad y la gestión privada de los recursos, esto es, recuperar la gestión directa de los servicios públicos que presta un municipio de manera indirecta (Vilar, 2006), de modo que ello traiga consigo beneficios tales como generación de empleos y ahorro de recursos financieros, entre otros.

Las acciones que ha puesto en práctica el sector privado han sido cuestionadas por los nuevos gobiernos que apuestan por la vuelta de los servicios públicos debido a que con el paso de los años se ha visto que la privatización no es la mejor forma de incentivar y aumentar las economías, por lo cual algunos de esos gobiernos han emprendido acciones que se inclinan a la remunicipalización, que puede llevarse a cabo, a decir de Tornos (2016), solo si se trata de actividades económicas que requieren servicios públicos que se encuentran en el mandato real, si el municipio asume la ejecución del servicio como una prestación efectiva y si el municipio asume el régimen de monopolio.

España es uno de los países que está a favor de la remunicipalización, ya que gracias a ella pueden participar los entes locales, los organismos autónomos locales, las entidades públicas locales y las sociedades mercantiles locales, 
con capital social íntegramente de titularidad pública (Vilar, 2006), y obtener beneficios directos en las comunidades receptoras.

\section{Conclusiones}

Si se tiene en cuenta que el agua se considera un recurso vital para la supervivencia del ser humano y de la naturaleza, pero sobre todo para el desarrollo de la actividad turística, este se encuentra en una presión creciente, gracias al crecimiento de la demanda de ese sector, que ha conducido a un crecimiento de la competencia y de los conflictos relacionados con el agua.

A lo largo de su historia Benidorm ha tenido problemas por abastecimiento que ha resuelto bajo distintos planes y acciones que le han permitido asegurar y garantizar el abasto del recurso. El problema es, sin embargo, más perverso, por parte del turismo, que acapara el agua como un recurso indispensable para satisfacer a los visitantes sin importar el costo social que esto implica para los pobladores locales.

La expansión de los grandes capitales a esta región a través del turismo da cuenta de la desposesión de los habitantes originarios de sus medios de reproducción y subsistencia, y de cómo se da libre paso a la mercantilización y los habitantes se vuelven únicamente trabajadores.

Esto demuestra que el sector privado es el beneficiado, y que los instrumentos de regulación solo sirven para legitimar que la distribución es equitativa cuando en la realidad no es así porque siempre existen argumentos y presiones para orientar a la sociedad a aceptar condiciones desfavorables.

Por lo tanto, la privatización del agua o del manejo del recurso no ha garantizado un buen funcionamiento ni de la actividad turística, ni de las actividades complementarias como la agricultura, ni ha logrado satisfacer las necesidades fundamentales de la población local. Entonces, la acumulación de capital es posible en la medida que logra articular con fines productivos la valorización de este destino a partir del territorio y del agua.

Por otro lado, con el fin de maximizar el bienestar social y económico, resultante de manera equitativa, sin comprometer la sostenibilidad de los ecosistemas vitales, se requieren políticas diseñadas por aquellos actores e instituciones que 
intervienen en el manejo de los recursos hídricos que a su vez tomen decisiones en todos los niveles, que beneficien principalmente a los grupos menos favorecidos y contemplen objetivos ambientales. Las nuevas propuestas que nacen de las legislaturas permiten visualizar un nuevo panorama de la gestión del agua en beneficio de las poblaciones.

Como ejemplo de ello, se puede considerar la llamada remunicipalización, con la que es posible regresar al sector público las gestiones privadas, en este caso la del agua, con la cual se generarían grandes beneficios, pero sobre todo se favorecería principalmente a las poblaciones locales. Al estar el agua en manos de la gestión pública, es más probable que los trabajadores tengan mayores oportunidades de crecimiento y, con ello, una mejor calidad de vida.

\section{Fuentes consultadas}

Agencia Europea de Medio Ambiente. (2016). Agencia Europea de Medio Ambiente. Unión Europea. Recuperado de http://www.eea.europa.eu/es/about-us/who

Amillo, F. (2013). El agua en Benidorm a través de su historia. Alicante. Recuperado de http://histobenidorm.blogspot.mx/2013/03/el-agua-en-benidorm-lolargo-de-su.html

Ayuntamiento de Benidorm. (2014). Benidorm en cifras. Alicante. Recuperado de www. benidormencifras.gob Candanedo, C. (1992). Conferencia Internacional sobre el Agua y el Medio Ambiente: Cuestiones de desarrollo para el siglo 21. Recuperado de http://es.ircwash.org/sites/default/files/71-ICWE92-19134. pdf

Comisión Europea. (2016). Dirección General de Medio Ambiente. Comisión Europea. Recuperado de http://hispagua.cedex.es/node/87533

Confederación Hidrográfica del Júcar. (2015). Plan Hidrológico de la Cuenca. Gobierno de España. Recuperado de http://www.chj.es/es-es/medioambiente/Paginas/Medio_ambiente.aspx

Consorcio de Agua de la Marina Baja. (2015). Consorcio de Agua de la Marina Baja. Recuperado de http://www.consorciomarinabaja.org/ [2015, 10 de julio]. Cordero, A. (2006). Nuevos ejes de acumulación y naturaleza. El caso del turismo. Buenos Aires: Consejo Latinoamericano de Ciencias Sociales.

Departamento de Asuntos Económicos y Sociales de Naciones Unidas. (2015). El agua es fuente de vida. Asamblea General de la Naciones Unidas. Recuperado de 
http://www.un.org/spanish/waterforlifedecade/human_right_to_water.shtml

Dotres, Y. (2003). ¿De qué manera el acelerado desarrollo tecnológico que se vive actualmente impacta en el sector turístico? Holguín: Universidad de Holguín.

Espinosa, A. (2011). La evolución del turismo en España. El ejemplo de Benidorm. Alicante: Departamento de Geografía Humana-Universidad de Alicante.

Gaviria, M. (1976). El turismo de invierno y el asentamiento de extran-jeros en la provincia de Alicante. Alicante: Instituto de Estudios Alicantinos.

Generalitat Valenciana. (2015). Turismo en la comunidad valenciana. Comunitat Valenciana. Recuperado de http://va.comunitat-valenciana.com/

Gil, A. y Rico, A. (2015). Consorcio de Agua de la Marina Baja. Gestión convenida, integral y sostenible del agua. Alicante: Instituto Interuniversitario de GeografíaUniversidad de Alicante.

Hall, D. (2011). Remunicipalización de los servicios municipales en Europa. Londres: Unidad Internacional de Investigación de Servicios Públicos-Universidad de Greenwich.

Harvey, D. (2005). El nuevo imperialismo. Nueva York: Oxford University Press.

Hiernaux, D. (2002). Imaginarios sociales y turismo sostenible. San José: Facultad Latinoamericana de Ciencias Sociales.

Higueras, M. (2012). Benidorm. Estudio de su modelo urbanístico (Proyecto de fin de grado en Ingeniería de Edificación). Universidad de Alicante, Alicante.

Instituto Nacional de Estadística. (2015). Datos de Benidorm. Recuperado de http:// www.ine.es/

Jouralev, A. (2003). Los municipios y la gestión de los recursos hídricos. Santiago: División de Recursos Naturales e Infraestructura-Comisión Económica para América Latina y el Caribe, Chile.

Juárez, C. (1994). Infraestructura hidráulica y crecimiento económico en la Marina Baja (Alicante). Universidad de Alicante. Sharq Al-Andalus 1993-1994 (10-11). Recuperado de: https://rua.ua.es/dspace/bitstream/10045/17692/1/Sharq\%20 Al-Andalus_10_11_28.pdf

Lengyel, E. (1997). Globalización, capital y estado. Recuperado de http://www.redalyc. org/pdf/267/26700819.pdf

López, A. y Marín, G. (2010). Turismo, capitalismo y producción de lo exótico: una perspectiva crítica para el estudio de la mercantili-zación del espacio y la cultura. Revista Relaciones Zamora, 31(123), 219-258.

Mazón, T. (2010). Benidorm. Un destino turístico de altura. Gran Tour: Revista de Investigaciones Turísticas, 2, 8-22. 
Mazón, T. y Delgado, E. (2009). Los discursos públicos de los agentes sociales de Benidorm. Alicante: Universidad de Alicante.

Mazón, T., Delgado, E. y Hurtado, J. (2011). El éxito de un destino turístico: el Benidorm de Mario Gaviria (Cátedra de Estudios Turísticos. Pedro Zaragoza Orts). Universidad de Alicante, Alicante.

Mendoza, J. (2000). ¿Cuál es el rol del Estado? Revista Facultad de Ciencias Económicas, $V(5), 45-62$.

Ministerio de Agricultura, Alimentación y Medio Ambiente. (2015). Ministerio de Agricultura, Alimentación y Medio Ambiente. Gobierno de España. Recuperado de http://www.magrama.gob.es/es/

Ministerio de Industria, Energía y Turismo. (2015). Balance año turístico, 2014. Recuperado de http://www.minetur.gob.es/es-es/gabinete prensa/notasprensa/2015/documents/150121dossier \% 20balance \% 20tur \% C3 \% ADstico \% 20 2014.pdf [2016, 14 de enero].

Observatorio Turístico de la Comunidad Valenciana. (2014). El turismo en la comunidad valenciana. Generalitat Valenciana. Recuperado de http://www.turisme.gva.es/turisme/es/files/pdf/esta distiquesdeturisme/anuarios/ TCV2014-def.pdf

Organización Mundial del Turismo. (2015). Panorama омт del turismo internacional. Recuperado de http://www.e-unwto.org/doi/pdf/10.18111/9789284416875 [2016, 18 de enero].

Ordoñez, Z. y Rovira, M. (2011). Benidorm, de la caña de pescar a la sombrilla. Alicante: Universidad de Alicante.

Organización de las Naciones Unidas. (2013). Turismo sostenible: contribución del turismo al crecimiento económico y al desarrollo sostenible. Conferencia de las Naciones Unidas sobre Comercio y Desarrollo. Autor.

Palafox, A. (2013). El turismo como eje de acumulación. Nómadas. Revista Crítica de Ciencias Sociales y Jurídicas, Núm. Especial: América Latina.

Palafox, A., Zizumbo, L. y Arriaga, E. (2010). El turismo como eje de acu-mulación: el caso del sector hotelero en México. Multiciencias, 10(2), 193-202.

Parlamento Europeo. (2016). Comisión de Medio Ambiente, Salud Pública y Seguridad Alimentaria. Parlamento Europeo. Recuperado de http://www.eppgroup. eu/es/ENVI [2016, 8 de junio].

Petrella, R. (2011). El manifiesto del agua. Barcelona: Icaria.

Rico, A. (2007). Tipología de consumo de agua en abastecimientos urbano-turísticos de la comunidad valenciana. Investigaciones Geográficas, 42, 5-34. 
Rico, A. (2010). Plan Hidrológico Nacional y Programa A.G.U.A.: Repercusión en las regiones de Murcia y Valencia. Investigaciones Geográficas, 51, 235-267.

Rico, A. y Baños, C. (2015). El ciclo hidrosocial en Benidorm: un modelo de gestión concentrado, integral y sostenible del agua. Seminario de Agua y Turismo. Instituto Interuniversitario de Geografía. Universidad de Alicante. Recuperado de://geografia.uab.cat/grats/seminari2015/04\%20Rico \% 20y \% 20Ba \% C3\% B1 os \% 20 ciclo\%20hidrosocial\%20benidorm.pdf]

Rico, A., Olcina, J. y Baños, C. (2014). Competencias por el uso del agua en la provincia de Alicante: experiencias de gestión en la armonización de usos urbanoturísticos y agrícolas. Documentos de Análisis Geográfico, 60(3), 523-548.

Romero, J., Toledo, Y. y Ocampo, V. (2014). El sector servicios: Revisión de los aportes para su teorización y estudio. Tesistecatl, 6(16). Recuperado de: http://www. eumed.net/rev/tecsistecatl/n16/sector-servicios.pdf.

Saladié, Ò. (2013). El agua: un recurso natural imprescindible (Módulos universitarios de desarrollo sostenible). Universidad Rovira i Virgili. Recuperado de http:// www.desenvolupamentsostenible.org/index.php?option = com_conte nt \&view $=$ article\&id $=4507 \&$ Itemid $=507 \&$ lang $=$ es

Sánchez, M., Rodríguez, N. y Salas, M. (2011). La gestión del agua en España. La unidad de Cuenca Water management in Spain. The basin unit. Estudios Regionales, 92, 199-220.

Seoane, J. (2013). Modelo extractivo y acumulación por despojo. En J. Seoane, E. Taddei y C. Algranati (coords.), Extravismo, despojo y crisis climática. Desafíos para los movimientos sociales y los proyectos emancipatorios de Nuestra América. Buenos Aires: Ediciones Herramienta / El Colectivo.

Tornos, J. (2016). La remunicipalización de los servicios públicos locales. Alicante. Recuperado de www.deje.ua.es/es/es/derecho-administrativo/documentos/ponencias-congreso-profesores-administrativos

Torregrosa, T. (2009). Modelo socioeconómico de la gestión de los recursos hídricos en la comarca de la marina baja (Alicante), un enfoque de gestión integrada de recursos hídricos (Tesis doctoral). Departamento de Análisis Económico Aplicado-Universidad de Alicante, Alicante.

Vera, J. (1986). Turismo y urbanización en el litoral Alicantino (Tesis doctoral). Sección de Geografía e Historia-Facultad de Filosofía y Letras-Universidad de Alicante, Alicante.

Vera, J. (2006). Agua y modelo de desarrollo turístico: la necesidad de nuevos criterios para la gestión de los recursos. Boletín de la A.G.E., 42, 155-178. 
Vilar, M. (2006). La Remunicipalización de los servicios públicos locales, a escena. Recuperado de www.bolinf.es/wp/archives/75950

Witker, J. (2015). El agua como recurso natural, desde la perspectiva del derecho económico. México: Instituto de Investigaciones Jurídicas-Universidad Nacional Autónoma de México.

Wollmann, H. (2013). La experiencia de los ordenamientos europeos: iun "retorno" a las gestiones públicas/municipales? Cuadernos de Derecho Local, 31, 70-80.

World Travel \& Tourism Council. (2015). Análisis del impacto económico. Recuperado de http://sp.wttc.org/research/economic-research/economic-impactanalysis 
